\title{
Depression - public health perspective
}

\begin{abstract}
Depression is a leading cause of disability affecting 400 million people of all ages worldwide. It is estimated that with current trends of demographic and epidemiological transition, by 2020, it would be the second leading cause of disability adjusted life years. In its severe form it can be a cause of suicide. Persons suffering from major depression and schizophrenia also have $40 \%$ - $60 \%$ higher risk of dying prematurely than general population. Unfortunately, only fewer than half of those affected in the world receive treatments. In some countries the figure is as low as $10 \%$. Common barrier to effective care include lack of resources, lack of trained health care providers, social stigma etc. Keeping in view the gravity of problem, World Health Organization has chosen depression as a theme for World Health Day 2017 ("Depression: let's talk"). Also, Comprehensive Global Action Plan 2013-20 and WHO's Mental Health Gap Action Programme (mhGAP) provide road map for promoting mental well being, preventing mental disorders. At national level, in India, National Mental Health Programme (NMHP) envisages promotion of mental health to prevent depression. But still, effective implementation of strategies is the need of the hour.
\end{abstract}

Keywords: Depression, public, health, perspective
Volume 7 Issue 6 - 2018

\section{Kanwal Preet Gill}

Department of Community Medicine, Sri Guru Ram Das Institute of Medical Sc. \& Research, India

Correspondence: Kanwal Preet Gill, Associate Professor, Department of Community Medicine, Sri Guru Ram Das Institute of Medical Sc. \& Research, Amritsar, India, Tel: 919464702373,Email kpraet224@gmail.com

Received: February 02, 2017| Published: November 20, 2018

\section{Introduction}

Depression is a leading cause of disability affecting 400 million people of all ages worldwide. ${ }^{1}$ It accounts for $4.3 \%$ of the global burden of disease and is estimated that with current trends of demographic and epidemiological transition, by 2020, it would be the second leading cause of disability adjusted life years. ${ }^{2}$ Depression is an illness characterized by persistent sadness and a loss of interest in activities one normally enjoys, accompanied by an inability to carry out daily activities, for at least two weeks. ${ }^{3}$ In its severe form it can be a cause of suicide. Worldwide, suicide is the second leading cause of death in 15-29-year-olds. An estimated 804,000 suicide deaths occurred globally in 2012, accounting for an annual global agestandardized suicide rate of 11.4 per 100000 population. ${ }^{4}$ There are inter-relationship between depression and physical health. Persons suffering from major depression and schizophrenia have 40\%-60\% higher risk of dying prematurely than general population. ${ }^{2}$ Depression predisposes a person to diabetes and myocardial infarction. Both these diseases conversely lead to depression. Similarly many risk factors like socioeconomic status, alcohol use and stress are common to both mental disorders and non-communicable diseases. Women are more affected in comparison to men. Adolescents, middle aged women, elderly, unmarried or singles, people from lower socioeconomic status, people uprooted from their natural habitat are some of the vulnerable groups. Though these vulnerable groups in society differ across countries, but in general they share common challenges related to their social and economic status, social supports, and living conditions which includes:

I. Stigma and discrimination

II. Violence and abuse,

III. Restrictions in exercising civil and political rights

IV. Exclusion from participating fully in society

V. Exposed to maltreatment and neglect
VI. Lack of educational, job and vocational opportunities

VII. Reduced access to health and social services

VIII.Reduced access to emergency relief services

IX. Increased disability and premature death.

Despite the fact that the burden of depression and other mental disorders is increasing in all countries of the world with great social as well as economic consequences, the countries are still somewhat reluctant to invest in the mental health of its citizens. Unfortunately only fewer than half of those affected in the world receive such treatments. In some countries the figure is as low as $10 \% .{ }^{5}$ Common barrier to effective care include lack of resources, lack of trained health care providers, social stigma etc.

Keeping in view the gravity of problem, World Health Organization has chosen depression as a theme for World Health Day 2017 ("Depression: let's talk"). Also, Comprehensive Global Action Plan 2013-20 provides road map for promoting mental well being, preventing mental disorders and reducing the mortality, morbidity and disability. It focuses on strengthening effective leadership, providing comprehensive, integrated and responsive health and social care services in community settings. It also provides guidance for implementing evidence based strategies and strengthening information systems for mental health. It relies on six important principles and approaches i.e. universal health coverage, human rights, evidence based practice, life course approach, multisectoral approach and empowerment of persons with mental health disorders and psychosocial disabilities. The Programme asserts that with proper care, psychosocial assistance and medication, tens of millions of people with mental disorders, including depression, could begin to lead normal lives - even where resources are scarce.

Depression is one of the priority conditions covered by WHO's Mental Health Gap Action Programme (mhGAP). It provides evidence based guidance, tools and training packages to improve the 
mental health care services in different countries, especially countries with poor settings. It focuses on integrated approach for priority conditions as well as capacity building through non-specialized health care providers. Psychological treatment in the form of behavioral activation, cognitive behaviour therapy (CBT) and interpersonal therapy is the recommended first line therapy recommended by World Health Organization. Group Interpersonal Therapy (IPT) may be provided at health-care centers by supervised non-specialized staff with dedicated time to deliver the intervention. Where such staffs are not available at the centre, non-specialized health-care providers may refer for IPT delivered in community settings, within social services or through specialized mental health care. ${ }^{6}$

At national level, in India, National Mental Health Programme (NMHP) was started in 1982 to ensure the availability and accessibility of minimum mental healthcare for all. On the basis of "Bellary Model", the District Mental Health Programme was launched in IX Five Year Plan (1996). During X Five Year Plan (2003), NMHP was re-strategized with Modernization of State Run Mental Hospitals, Manpower Development Schemes and Up gradation of Psychiatric Wings of Medical Colleges/General Hospitals. NMHP also envisages health promotion of adolescents through life skills approach. ${ }^{7}$ Although community based services are widely regarded as the nest approach for providing mental health treatment and care, most low and middle income countries continue to spend the vast majority of their scarce mental health resources managing people with mental disorders in mental hospitals. World Health Organization strongly recommends 'Deinstitutionalization' with five principles which are:

1) Community based services must be in place

2) Heath work force must be committed to change

3) Political support at the highest and the broadest level is crucial

4) Timing is the key

5) Additional financial resources are needed
For this, if on one hand scaling up of existing health services is required, on the other hand transformation of health system to implement evidence based approaches is needed. ${ }^{1}$ For better integration, action is required not only within the health sector but intersectoral co-ordination for implementation of effective and efficient strategies for creating awareness, preventing depression and promoting mental health is the need of the hour. World Health Organization's one year campaign for creating awareness among masses is a good step.

\section{Acknowledgements}

None.

\section{Conflict of interest}

The author has no conflicts of interests in this work.

\section{References}

1. World Health Organization. Integrating the response to mental disorders and other chronic diseases in health care system. 2014.

2. Lopez AD, Mathers CD, Ezzati M, et al. Global Burden of Disease and Risk Factors. Washington: The World Bank; 2006.

3. World Health Organization. Depression: What you should know. 20162017.

4. World Health Organization. Methods and data sources for global causes of death 2000-2012. 2014.

5. WHO. Fact Sheet.

6. World Health Organization. Group interpersonal therapy for depression. 2016.

7. Kishore J. National Mental Health Program. $9^{\text {th }}$ edn. New Delhi: Century Publications; 2007. 